\title{
Experimental Comparison of Outdoor Baseline Measurements by Different Methods
}

\author{
Youyi Gu ${ }^{1}$, Li Wang ${ }^{1}$, Fengzhuo Xiang ${ }^{2}$, Wen Ouyang ${ }^{1}$ and Lixing Jiang ${ }^{1, *}$ \\ ${ }^{1}$ Information Engineering University, Zhengzhou, Henan, 450001, China \\ ${ }^{2} 61287$ Troop, Chengdu, Sichuan, 610036, China
}

\begin{abstract}
Outdoor baseline is the special length standard in the field of surveying and mapping, it can be used to verify the addition and multiplication constants of the total station and other photoelectric rangefinders. In order to ensure the authenticity, accuracy and reliability of verification results, conducting outdoor baseline traceability periodically is essential. At present, direct measurement by $24 \mathrm{~m}$ invar tape or high precision electro-optical measurement is mainly used to achieve the traceability of outdoor baseline in China. Based on Shenyang baseline field, high precision rangefinder $\mu$-base, $24 \mathrm{~m}$ invar tape and high precision GNSS receivers are used for comparison experiments, and the experimental results are analyzed.
\end{abstract}

\section{Introduction}

At present, there are three calibration methods to measure the standard length of outdoor baseline, optical interferometry by Vaisala interference comparator, direct measurement by $24 \mathrm{~m}$ invar tape and high precision electro-optical measurement.

Optical interferometry by Vaisala interference comparator is the most accurate method to calibrate baseline in the world. With the help of the Finnish geodetic research institute, based on the experience of building the Nummela baseline, there were built $432 \mathrm{~m}$, $576 \mathrm{~m}$ and $378 \mathrm{~m}$ baselines in Changyang, Liquan and Chengdu respectively. Due to urban construction, Wenchuan earthquake and other factors, those baselines accuracy was low, and now it has been abandoned.

The first geodetic survey team is the only national field large-length quantity transmission institution authorized by the state, and undertakes the periodic verification work of dozens of outdoor baselines. The measurement method by $24 \mathrm{~m}$ invar tape is intuitive, and the error correction model is clear, but the measurement efficiency is low. In the field, the measurement will also be affected by temperature, tension, wind speed and other factors. If the baseline is long, the cumulative error generated by piecewise measurement will increase.

In terms of photoelectric ranging, $\mu$-base, a new generation of high-precision range finder launched in 2012, is convenient to carry, simple to operate, and has good performance. After model correction of meteorological parameters, the instrument can achieve high accuracy, which can be used for outdoor baseline calibration.

In China, the method by $24 \mathrm{~m}$ invar tape or highprecision photoelectric rangefinder is mainly used to calibrate outdoor baseline. A large number of experimental facts have shown that the transmission of quantity value of outdoor baseline is still inconsistent with that in foreign countries. Through the unremitting efforts of researchers, the systematic error is gradually reduced. Compared with those two methods, the measurement baseline accuracy of high-precision GNSS receiver is lower, which is not in line with the relevant requirements of outdoor baseline long measurement transmission system in China.

Through the outdoor baseline consistency comparison experiment, this paper also conducted an indepth study on the measurement of high-precision GNSS receiver, in order to continuously improve the length measurement value transfer system for beneficial exploration.

\section{Comparing experiment}

\subsection{Measuring method by $\mu$-base rangefinder}

On the afternoon of July 31, 2019, in Shenyang outdoor baseline, the measurement was made by using $\mu$-base rangefinder. Two sets of ventilation meteorological sensors were used to measure meteorological elements by "two-point method", and the meteorological elements were input into Pilot software for ranging correction in real time. In order to ensure the ranging accuracy, ADM signal strength should not be lower than 150. It is often considered that the effective ranging range of $\mu$-base is $1.5 \sim 160 \mathrm{~m}$. Under favorable weather conditions such as cloudy days and no wind, the maximum measuring distance can exceed $200 \mathrm{~m}$. The setting of the observation scheme is shown in figure 1.

\footnotetext{
* Corresponding author: 1943624866@qq.com
} 


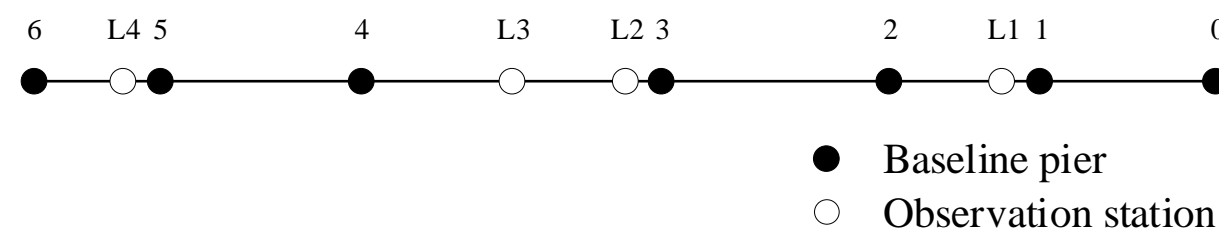

Fig. 1. Schematic diagram of $\mu$-base rangefinder observation.

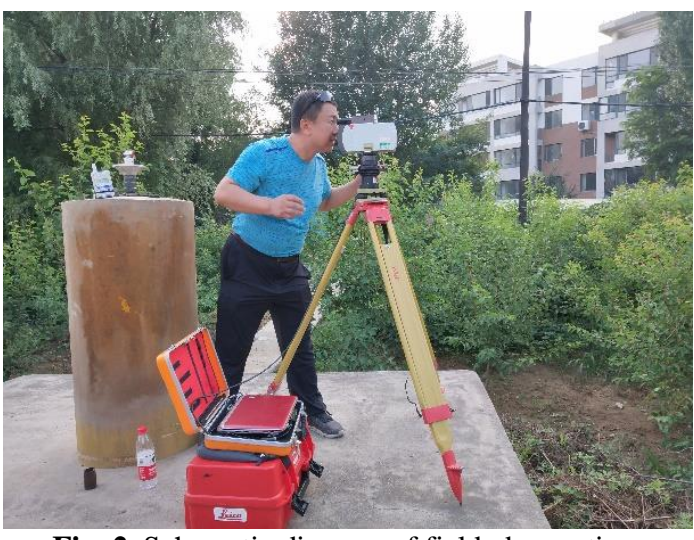

Fig. 2. Schematic diagram of field observation.

In the whole process from the beginning to the end of the measurement, the temperature change and wind speed were small, the illumination was not strong, $\mu$ -

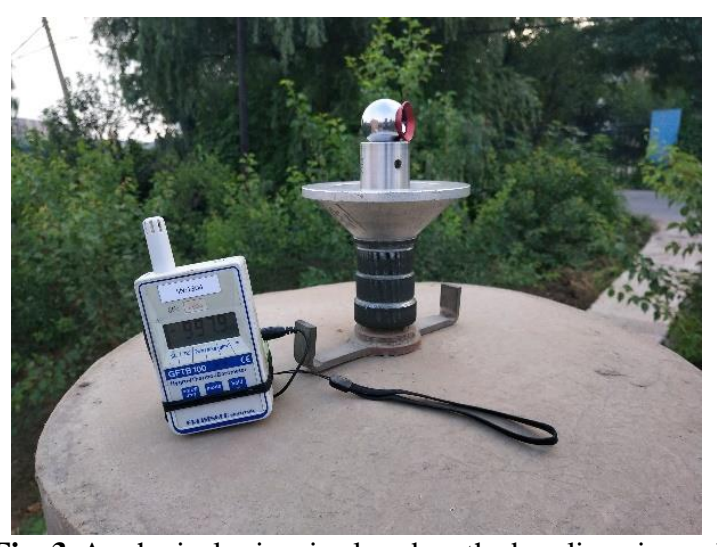

Fig. 3. A spherical prism is placed on the baseline pier and meteorological elements are measured by a ventilated weather sensor.

base rangefinder reading had a good repeatability. The original measurement data is shown in table 1.

Table 1. Raw data results of measurement.

\begin{tabular}{|c|c|c|c|c|c|c|}
\hline $\begin{array}{c}\text { Measurement } \\
\text { route }\end{array}$ & \multicolumn{3}{|c|}{ Slope distance of $\mu$-base/m } & $\begin{array}{c}\text { Atmospheric } \\
\text { pressure/ hPa }\end{array}$ & Temperature/ ${ }^{\circ} \mathrm{C}$ & Humidity/\% \\
\hline L1-0 & 169083.983 & 169083.986 & 169083.985 & 997.8 & 30.8 & 70 \\
\hline L1-1 & 1073.270 & 1073.270 & 1073.270 & 997.8 & 30.8 & 70 \\
\hline L1-2 & 143027.575 & 143027.580 & 143027.585 & 997.8 & 30.8 & 70 \\
\hline L2-2 & 217060.710 & 217060.703 & 217060.698 & 997.7 & 30.7 & 72 \\
\hline L2-3 & 1059.268 & 1059.269 & 1059.265 & 997.7 & 30.7 & 72 \\
\hline L3-3 & 143281.852 & 143281.844 & 143281.842 & 997.8 & 30.3 & 74 \\
\hline L3-4 & 144751.863 & 144751.857 & 144751.854 & 997.8 & 30.3 & 74 \\
\hline L4-4 & 193170.159 & 193170.148 & 193170.191 & 997.8 & 30.2 & 73 \\
\hline L4-5 & 1112.710 & 1112.704 & 1112.700 & 997.8 & 30.2 & 73 \\
\hline L4-6 & 94895.974 & 94895.972 & 94895.976 & 997.8 & 30.2 & 73 \\
\hline
\end{tabular}

On August 2, the first geodetic survey team used baseline piers. Horizontal distance results are obtained electronic level to measure the height difference of the

by using Pythagorean theorem, as shown in table 2.

Table 2. Calculation results of horizontal distance.

\begin{tabular}{|c|c|c|c|}
\hline Measurement route & Height difference $/ \mathbf{m}$ & Slope distance of $\boldsymbol{\mu}$-base $/ \mathbf{m}$ & Slope distance of $\boldsymbol{\mu}$-base $/ \mathbf{m}$ \\
\hline $0-1$ & -0.0655 & 168.01071 & 168.01070 \\
\hline $1-2$ & 0.0705 & 144.10085 & 144.10083 \\
\hline $2-3$ & 0.258 & 216.00144 & 216.00129 \\
\hline
\end{tabular}




\begin{tabular}{|c|c|c|c|}
\hline $3-4$ & 0.163 & 288.0337 & 288.03365 \\
\hline $4-5$ & 0.2185 & 192.05746 & 192.05734 \\
\hline $5-6$ & 0.1265 & 96.00868 & 96.00860 \\
\hline
\end{tabular}

\subsection{Static measurement by GNSS receivers}

On August 1, static measurement experiment was carried out. Three Trimble NetR9 receivers were set up at baseline piers 3, 5 and 6 with a wide field of view, the antenna was uniformly oriented to the north, the sampling interval was 10s, the height cut-off Angle was $10^{\circ}$, and the measurement time was 24 hours. Before starting up the receivers, measure the antenna height, as shown in figure 4 , and the field diagram of measurement is shown in figure 5 .

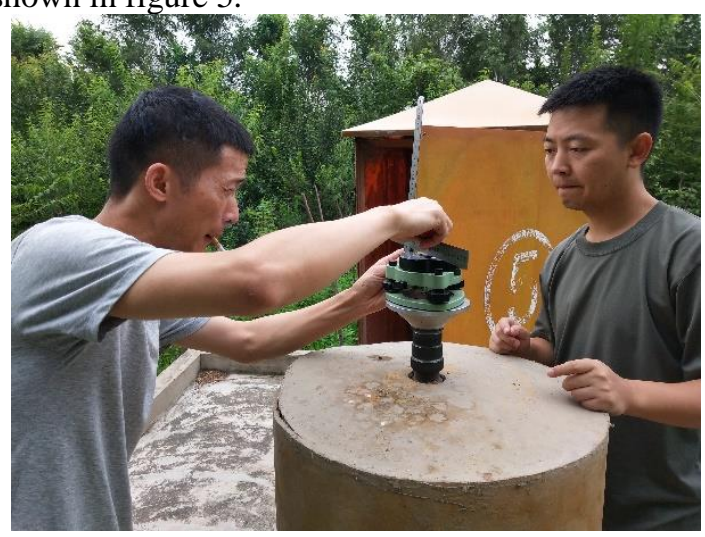

Fig. 4. Measure the height of the instrument from three directions and find an average value.

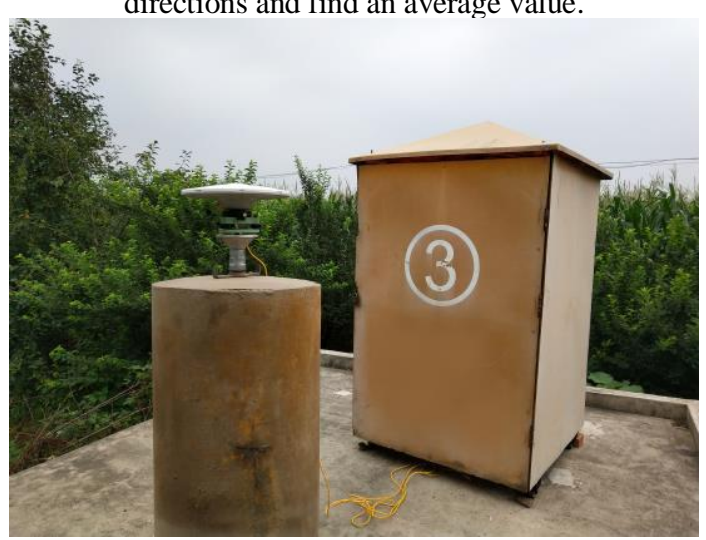

Fig. 5. A Trimble NetR9 receiver was set up at baseline piers 3 for static measurement experiment.

We select Trimble DL mobile application to send raw data files to $\mathrm{PC}$ via bluetooth communication. GAMIT 10.5 software is used for baseline calculation, it runs on the LINUX operating system. Data preparation is required before calculation. Convert the original observation file of Trimble too file by Convert To RINEX program. And download the corresponding precise ephemeris SP3 file, navigation message file and table files that need to be updated. files.

The most critical of these is to configure four table

- Station approximate coordinate file. Based on the research of Liu Yangyang, station approximate coordinate file is generated by using the results of single point positioning solution.

- Station information file. Enter the correct parameters manually, such as antenna height, antenna type, antenna high measurement mode, receiver software version number, etc.

- Station constraint table. We chose the short sittbl, the most important thing is to set $3 \mathrm{~d}$ coordinate constraint, generally, it is set as the 2-3 times median error of coordinate accuracy. Considering the accuracy of single point positioning measurement, 3d coordinate constraints are all set to $9.999 \mathrm{~m}$.

- Data processing parameter setting table. Some parameters are set as follows, Choice of Experiment $=$ RELAX, Type of Analysis $=1$ ITER, Choice of Observable $=$ LC_AUTCLN, Zenith Delay Estimation $=$ Y, Interval zen $=1$, Tides applied $=31$, Elevation Cutoff $=15$.

After the file preparation and configuration is completed, enter the commands in turn according to the GAMIT baseline calculation process. Table 3 shows the results of baseline calculation and horizontal distance.

Table 3. Font styles for a reference to a journal article.

\begin{tabular}{|c|c|c|}
\hline $\begin{array}{c}\text { Measurement } \\
\text { route }\end{array}$ & $\begin{array}{c}\text { Slope distance } \\
\text { /m }\end{array}$ & $\begin{array}{c}\text { Horizontal } \\
\text { distance } / \mathbf{m}\end{array}$ \\
\hline $5-6$ & 96.0125 & 96.01242 \\
\hline $3-5$ & 480.0874 & 480.08725 \\
\hline $3-6$ & 576.0999 & 576.09968 \\
\hline
\end{tabular}

In the output o file, standard root mean square NRMS is an evaluation index of baseline solution accuracy. The smaller the value of NRMS is, the higher the solution accuracy is. The value of NRMS in this experiment is 0.25 , indicating that the solution result is reliable.

\subsection{Direct measurement by $24 \mathrm{~m}$ invar tape}

From August 2 to 3, the first geodetic survey team measured the baseline by $24 \mathrm{~m}$ invar tape. The measurement process is shown in figure 6 and 7.

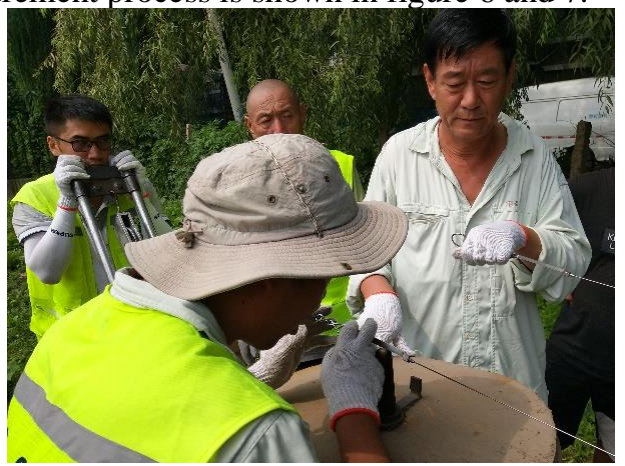

Fig. 6. The members of the first geodetic survey team cooperate closely to measure the baseline. 


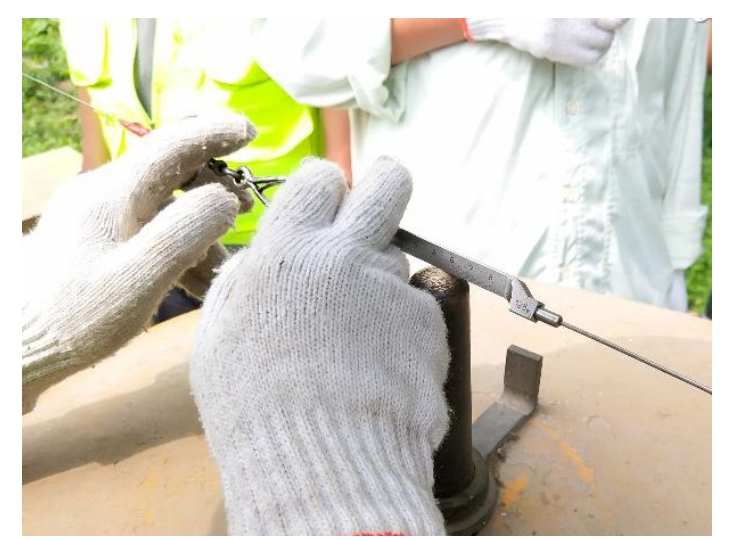

Fig. 7. When measuring, the surveyor takes a reading of the moment. The $24 \mathrm{~m}$ invar tape has mm marks on both ends less than $8 \mathrm{~cm}$.

They used a tape measure to divide the baseline into three sections of equal distance, and used four $24 \mathrm{~m}$ invar tapes with different expansion coefficients to carry out sub-group measurement. In each section, two went to the measurement and two went back to the measurement.

The measurement results of each ruler were corrected by temperature correction, ruler length correction and tilt correction, and the measurement results of 4 rulers were averaged. The whole length of the baseline was the sum of the lengths of each segment.

The measurement results are shown in table 4 .
Table 4. Measurement results of the $24 \mathrm{~m}$ invar tape.

\begin{tabular}{|c|c|}
\hline Measurement route & Horizontal distance/m \\
\hline $0-1$ & 168.01104 \\
\hline $1-2$ & 144.10073 \\
\hline $2-3$ & 216.00097 \\
\hline $3-4$ & 288.03432 \\
\hline $4-5$ & 192.05703 \\
\hline $5-6$ & 96.00871 \\
\hline
\end{tabular}

\section{Conclusions}

The comparison results are shown in table 5 and 6 .

According to table 5, the system error between $\mu$ base and $24 \mathrm{~m}$ invar tape is submillimeter, but it can not be ignored for the accuracy of baseline calibration. According to the preliminary experimental results, the error compensation algorithm and calculation model should be further optimized. For the GNSS receiver measurement method, there are several tall trees on the side of point 5 , which results in the accuracy of the two baselines involved in the calculation is damaged. In the next step, the comparison experiment between $\mu$-base and GNSS receiver can be carried out in open areas to reduce the influence of multipath effect.

Table 5. Results of consistency comparison between $\mu$-base and $24 \mathrm{~m}$ invar tape.

\begin{tabular}{|c|c|c|c|c|}
\hline \multirow{2}{*}{$\begin{array}{c}\text { Measurement } \\
\text { route }\end{array}$} & $\boldsymbol{\mu}$-base/m & Invar tape/m & $\mathbf{2}$ & \multicolumn{2}{|c|}{ System error } \\
\cline { 3 - 5 } & & & $\mathbf{m m} / \mathbf{k m}$ \\
\hline $0-1$ & 168.01070 & 168.01104 & -0.343 & -2.0 \\
\hline $1-2$ & 144.10083 & 144.10073 & 0.103 & 0.7 \\
\hline $2-3$ & 216.00129 & 216.00097 & 0.316 & 1.5 \\
\hline $3-4$ & 288.03365 & 288.03432 & -0.666 & -2.3 \\
\hline $4-5$ & 192.05734 & 192.05703 & 0.306 & 1.6 \\
\hline $5-6$ & 96.00860 & 96.00871 & -0.113 & -1.2 \\
\hline
\end{tabular}


Table 6. Comparison results of three measurement methods.

\begin{tabular}{|c|c|c|c|c|c|c|}
\hline \multirow{2}{*}{$\begin{array}{l}\text { Measurement } \\
\text { route }\end{array}$} & \multirow{2}{*}{$\begin{array}{l}\text { Invar } \\
\text { tape/m }\end{array}$} & \multirow{2}{*}{$\mu$-base/m } & \multirow{2}{*}{ GNSS/m } & \multicolumn{3}{|c|}{ System error/ mm } \\
\hline & & & & $1-2$ & $1-3$ & $2-3$ \\
\hline $3-5$ & 480.09135 & 480.09099 & 480.08725 & 0.36 & 4.10 & 3.74 \\
\hline $3-6$ & 576.10006 & 576.09959 & 576.09968 & 0.47 & 0.38 & -0.09 \\
\hline $5-6$ & 96.00871 & 96.00860 & 96.01242 & 0.11 & -3.71 & -3.82 \\
\hline
\end{tabular}

Outdoor baseline precision ranging will play a crucial role in the verification of precision measuring instruments, it is beneficial to the progress of length measurement system in China and the development of basic areas, it can play an important role in national defense construction, major infrastructure construction, aerospace, equipment support, national economy and other fields.

\section{Acknowledgments}

This research is funded by National key R\&D Program of China (2017YFF0206000), authors thank Senior engineer Fan Gang and Teacher Jia Zhen for their help.

\section{References}

1. Bozic, B., Huaan Fan, and Z. Milosavljevic. "Establishment of the MGI EDM calibration baseline." Survey Review 45.331 (2013): 263-268.

2. Rüeger, Jean M. "Correspondence on 'Establishment of the MGI EDM calibration baseline'by B. Bozic, H. Fan and Z. Milosavljevic." Survey Review 45.333 (2013): 474-476.

3. Pollinger, F., et al. "Metrology for long distance surveying: a joint attempt to improve traceability of long distance measurements." IAG 150 Years. Springer, Cham, 2015. 651-656.

4. Jokela, Jorma, and Pasi Häkli. Interference measurements of the Nummela Standard Baseline in 2005 and 2007. Finnish Geodetic Institute, 2010.

5. Jokela, Jorma, and Pasi Häkli. "Current research and development at the Nummela Standard Baseline. Conference proceedings." Shaping the Change, XXIII FIG Congress. 2006.

6. Li J.S., Liang P., Zhao Y., et al. (2008) Reliability of the Value of an Outdoor Large Dimension Standard-24m Invar Wire. Acta Metrologica Sinica, 29: 74-76.

7. Fu Z.A., Zhu J., Sun F.F., et al. (2011) Discussion about Traceability of Length Baseline. Journal of Geomatics Science and Technology, 28: 157-160.

8. Li G.Y., Fan B.X. (2017) The Development of Precise Engineering Surveying Technology. Acta Geodaetica et Cartographica Sinica, 46: 1742-1751.

9. Bao H., Zhao D.M., Wang R.P., et al. (2014) Baseline Field Experiments for the High Accuracy Baseline Verification Instrument $\mu$-base. Acta Metrologica Sinica, 35: 109-112.
10. Pollinger F., Meyer T., Beyer J., et al. (2012) The upgraded PTB $600 \mathrm{~m}$ baseline: a high-accuracy reference for the calibration and the development of long distance measurement devices. Measurement Science \& Technology, 23: 2910-2916. 